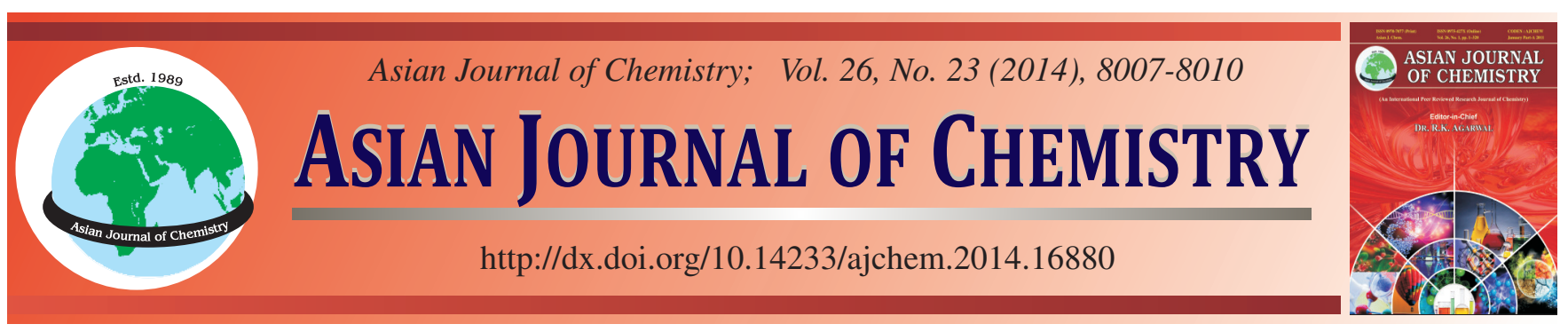

\title{
Simultaneous Determination of Serotonin, $\beta$-Hexosaminidase and Histamine in Allergic Guinea Pigs Caused by Three Herbal Injections
}

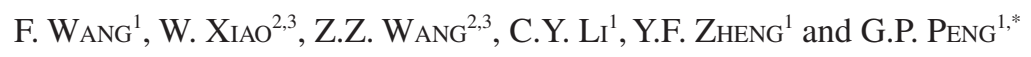

${ }^{1}$ College of Pharmacy, Nanjing University of Chinese Medicine, Nanjing 210023 P.R. China

${ }^{2}$ State Key Laboratory of New-Tech for Chinese Medicine Pharmaceutical Process, Jiangsu Kanion Pharmaceutical Co., Lianyungang 222001, Jiangsu, P.R. China

${ }^{3}$ Jiangsu Kangyuan Pharmaceutical Company Limited, Lianyungang, P.R. China

*Corresponding author: Tel/Fax: +86 25 86798186; E-mail: guopingpeng1@163.com

Received: 15 December 2013;

Accepted: 25 March 2014;

Published online: 15 November 2014;

AJC-16282

\begin{abstract}
The function of herbal injection is irreplaceable because of the quick action and definite therapeutic effects in the clinic, however allergic reactions frequently occurred and the fundamental research on its security is defective. In this study, in order to evaluate the allergenicity of Shuanghuanglian, Yinzhihuang and Danshen injection, in which two of them were often reported allergic reactions in the clinic and to explore the potential reasons of the allergenicity. Serotonin, $\beta$-hexosaminidase and histamine were used as the indexes to evaluate the allergenicity for the first time. Ovalbumin, chlorogenic acid and the three heberal injections were chosen to sensitize and challenge the guinea pigs. After administrated to stimulate the guinea pigs, concentrations of serotonin, $\beta$-hexosaminidase enzymes and histamine in Shuanghuanglian group, Yinzhihuang group and cholrogenic acid group were significantly increased compared with sodium chloride injection. However, there were no significant increases of three evaluated indexes in Danshen group. It is concluded that guinea pigs were more sensitized with Shuanghuanglian injection and Yinzhihuang injection which consists with the clinical medication. However, the sensitization of Danshen injection was very weak. The results have shown that using serotonin, $\beta$-hexosaminidase and histamine to evaluate the allergenicity of the herbal injection is reliable with high degree of accuracy. And cholrogenic acid might be one of the incentives of allergic reaction in Shuanghuanglian and Yinzhihuang injection.
\end{abstract}

Keywords: Herbal injection, Cholrogenic acid, Serotonin, $\beta$-Hexosaminidase, Histamine.

\section{INTRODUCTION}

Herbal medicines are widely used in many countries since ancient time ${ }^{1,2}$. Its excellent curative effect is still amazing the world. Herbal injection is a unique formulation of traditional Chinese medicine which was widely used in China. The function of herbal injection is irreplaceable in the clinical context because of the quick action and definite therapeutic effects ${ }^{3}$. However, the basic research on its security is defective, especially the sensitivity of allergy test. Over the past ten years, many severe allergic reactions of herbal injection had been reported in China which made great limits of its usage in the clinic $^{4,5}$. Allergic reaction is the most common adverse reaction of herbal injections ${ }^{6}$. Previous studies had identified two types of antibody-mediated systemic anaphylaxis, type I and type II is mediated by $\operatorname{IgE}$ and $\operatorname{IgG}$, respectively ${ }^{7-11}$. The allergic reaction of herbal injection is type I which mediated by IgE. Mast cells are the major effector cell of IgE mediated in allergic reaction and plays a key role in allergic reaction diseases ${ }^{12}$. When mast cells stimulated by the antigen, they may release active matters such as histamine, $\beta$-hexosaminidase and serotonin to produce a series of biological effects ${ }^{13,14}$. The existing studies mainly focused on one or two evaluation indexes such as $\operatorname{IgE}, \operatorname{IgM}, \beta$-hexosaminidase or histamine, which were lack of comprehensiveness ${ }^{15,16}$. Our previous experimental studies had suggested that serotonin (5-HT) could be detected quickly and had good correlation with the other allergy-related cytokines. In the present study, we used serotonin, $\beta$-hexosaminidase and histamine as indexes to evaluate the allergenicity of herbal injection for the first time.

\section{EXPERIMENTAL}

Male guinea pigs weighing 250-350 g were purchased from Nanjing Qinglongshan Animal Breeding Center. All animals were housed at $22{ }^{\circ} \mathrm{C}$ and $55 \pm 5 \%$ relative humidity. All experiments were carried out according to the guidelines of the Animal Care Committee of Nanjing University of Chinese Medicine.

Albumin chicken egg (OVA) and standard serotonin were purchased from Sigma (St Louis, USA). Cholrogenic acid was purchased from Kang Pharmaceutical Co., Ltd. (Jiangsu, 
China). Guinea pig $\beta$-hexosaminidase and histamine ELISA Kits were obtained from R\&D systems, Inc. Danshen injection was purchased from Dragon Pharmaceutical Co. Ltd. (Jiangsu, China). Shuanghuanglian injection was purchased from Zhenbao Pharmaceutical Co. Ltd. (Harbin, China). Yinzhihuang injection was purchased from Ley's Pharmaceutical Co.Ltd. (Changshu, China).

Waters 510 HPLC apparatus (Waters, USA), EC2000 Chem Station software (Elite Analytical Instruments Co. Ltd, Dalian), Waters 2645 electrochemical detector (Waters, USA), J2-MC high-speed refrigerated centrifuge (Beckman, USA), Phd200A Precision acidity meter (Broadcom Trade Co. Ltd., Shanghai), $0.45 \mu \mathrm{m}$ millipore filter, Hedera ODS-2 column $(150 \times 4.6$ $\mathrm{mm}, 5 \mu \mathrm{m})$.

Antigen sensitization and stimulation approach: Albumin chicken egg and cholrogenic acid was weighted accurately and dissolved in normal saline (NS) with final concentration of 5 and $0.5 \mathrm{mg} / \mathrm{mL}$, respectively. Sixty guinea pigs were divided into the following six groups ( $\mathrm{n}=10$ /group): normal saline group, Dansheng group, Shuanghuanglian group, Yinzhihuang group, cholrogenic acid group and albumin chicken egg group. Guinea pigs were exposed to all samples of $0.2 \mathrm{~mL}$ each time, QOD for a total of three subcutaneous administrations. After 16 days sensitization, chloral hydrate was dissolved in normal saline at the concentration of $10 \%$ and put guinea pigs under anesthesia. Animals were challenged by jugular vein injection. Anti-coagulant blood samples (sample A) were collected before anesthesia through the carotid artery. Anti-coagulant blood samples (sample B) were collected $0.5 \mathrm{~h}$ later after stimulation. Serum samples were obtained through centrifuged at $4000 \mathrm{r} / \mathrm{min}$ for $5 \mathrm{~min}$ and then stored at $-80{ }^{\circ} \mathrm{C}$.

Determination of serotonin: Equivalent methanol was added in serum samples and supernatants of the pretreated serums were collected after vortex for 30 s and centrifuged at $10,000 \mathrm{rpm}$ for $10 \mathrm{~min}$ for the detection of serotonin. An aliquot of $20 \mu \mathrm{L}$ sample solution was injected into the HPLC system. Analysis was performed on a Waters 510 HPLC apparatus coupled with Waters 2645 electrochemical detector and EC2000 ChemStation software. The separation was performed on a hedera ODS- $2 \mathrm{C}_{18}$ column. The mobile phase was composed of $25 \mathrm{mmol} / \mathrm{L}$ sodium dihydrogen phosphate (with 0.5 $\mathrm{mmol} / \mathrm{L}$ EDTA-Na and $3 \mathrm{mmol} / \mathrm{L}$ sodium heptanesulfonate, $\mathrm{pH}=4.6)$ : acetonitrile $(85: 15)$ at a flow rate of $0.8 \mathrm{~mL} / \mathrm{min}$. The column temperature was maintained at $25^{\circ} \mathrm{C}$.

IgE and $\boldsymbol{\beta}$-hexosaminidase determination: $\operatorname{IgE}$ and $\beta$ hexosaminidase in the serum samples were determined using enzyme-linked immunosorbent assay kits following the instruction of manufacturer. Standardized curves of IgE and $\beta$-hexosaminidase were shown in Table-1. The average increase rate of $\operatorname{IgE}$ and $\beta$-hexosaminidase were calculated according to follow formula:

$$
\text { Sample }(\%)=\frac{(\text { value } \mathrm{B}-\text { value } \mathrm{A})}{\text { value } \mathrm{A}} \times 100 \%
$$

Statistical analysis: Statistical significance was analyzed using t-test. The level of $\mathrm{P}<0.05$ and $\mathrm{p}<0.01$ and $\mathrm{P}<0.001$ was considered to be statistically significant.
TABLE-1

CALIBRATION CURVES FOR THE 3 INDEXES DETERMINED

\begin{tabular}{cccc}
\hline Index & Calibration curve & $\mathrm{R}^{2}$ & $\begin{array}{c}\text { Liner range } \\
(\mathrm{ng} / \mathrm{mL})\end{array}$ \\
\hline 5-HT & $\mathrm{y}=0.5661 \mathrm{x}+1.9108^{\mathrm{a}}$ & 0.998 & $30-960$ \\
$\beta$-hexosaminidase & $\mathrm{y}=0.0174 \mathrm{x}+0.0997^{\mathrm{b}}$ & 0.999 & $0-320$ \\
Histamine & $\mathrm{y}=0.0252 \mathrm{x}+0.1882^{\mathrm{c}}$ & 0.993 & $0-240$ \\
\hline${ }^{\mathrm{a}} \mathrm{y}$ represents the peak area and $x$ represents the concentration of \\
seroton in, ${ }^{\mathrm{b}} \mathrm{y}$ represents the optical density and $x$ represents the \\
$\begin{array}{l}\text { concentration of } \beta \text {-hexosaminidase, }{ }^{\mathrm{c}} \text { y represents the optical density } \\
\text { and } x \text { represents the concentration of histamine }\end{array}$
\end{tabular}

\section{RESULTS AND DISCUSSION}

Determination of 5-HT in allergic guinea pig plasma: The chromatogram of serotonin was shown in Fig 1. The chromatographic peak appeared quickly at around $12 \mathrm{~min}$ after the sample introduction. The linear range was 30-960 ng/mL and the limit of detection was $20 \mathrm{ng} / \mathrm{mL}$ (Table-1).

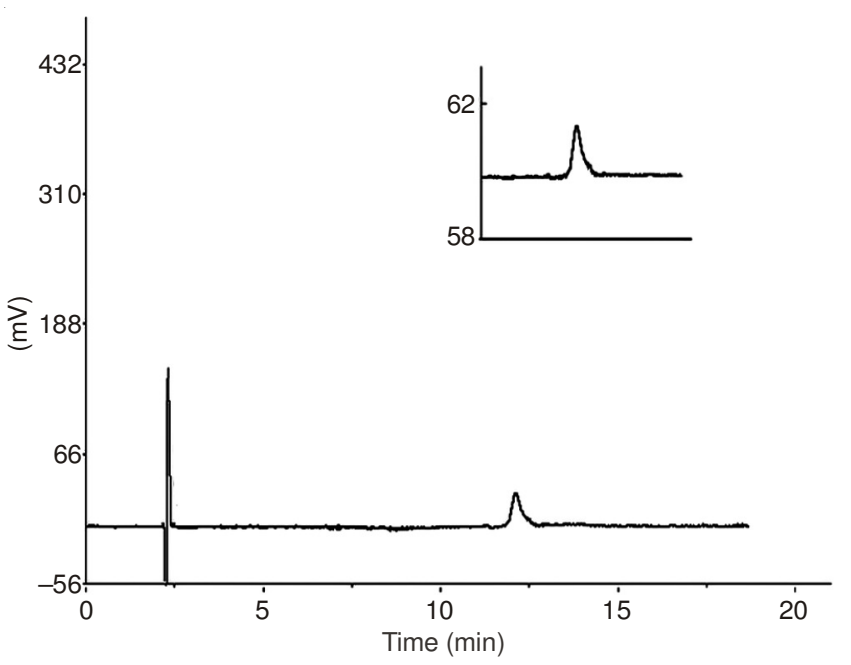

Fig.1. Chromatogram of serotonin

Animals were sensitized with normal saline, albumin chicken egg, Shuanghuan-glian injection, Yinzhihuang injection, Dansheng injection and chlorogenic acid through a subcutaneous way. After $30 \mathrm{~min}$, blood samples of the animals were collected for the analysis. As shown in Fig. 2, the concentration of 5-HT increased significantly in albumin chicken egg group, Shuanghuan-glian group, Yinzhihuang group and chlorogenic acid group compared with saline group. However, the increasing rate of 5-HT in Dansheng group was not statistically significant.

Determination of $\boldsymbol{\beta}$-hexosaminidase in allergic guinea pig plasma: Albumin chicken egg, Shuanghuanglian, Yinzhihuang, Dansheng and chlorogenic acid were administrated to challenge animals through a subcutaneous way. Sample A and sample B were collected for the analysis, in which $\beta$-hexosaminidase was measured with ELISA.

Compared with normal saline group, the increasing rate of $\beta$-hexosaminidase in albumin chicken egg group, Shuanghuanglian group, Yinzhihuang group and chlorogenic acid group were statistically significant. However, the increasing rate of $\beta$-hexosaminidase in Dansheng group was not statistically significant. The results were shown in Fig. 3. 


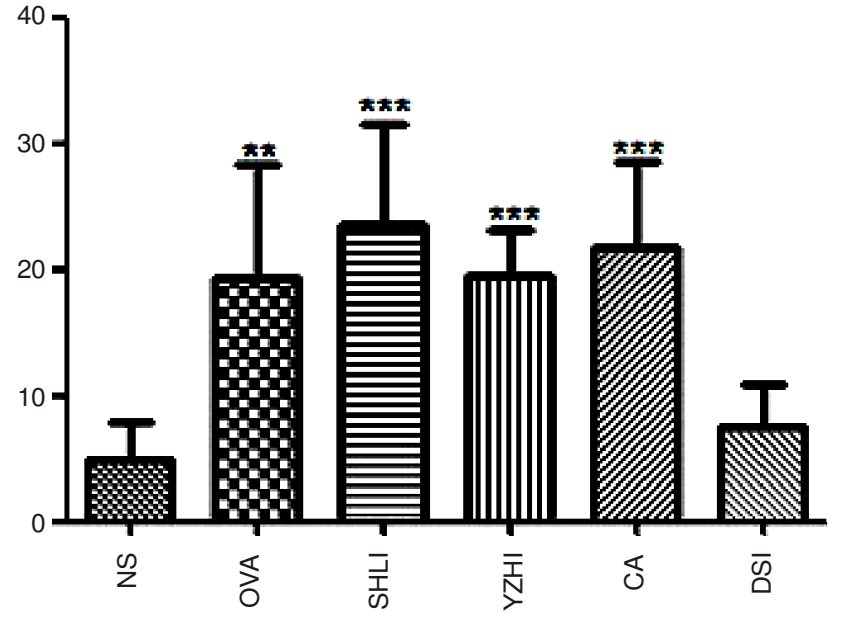

Fig. 2. Rising rate of 5-HT in allergic guinea pig plasma. Results are expressed as mean $\pm \mathrm{SD}(\mathrm{n}=6), * * \mathrm{P}<0.01, * * * \mathrm{P}<0.001$ vs. NS group

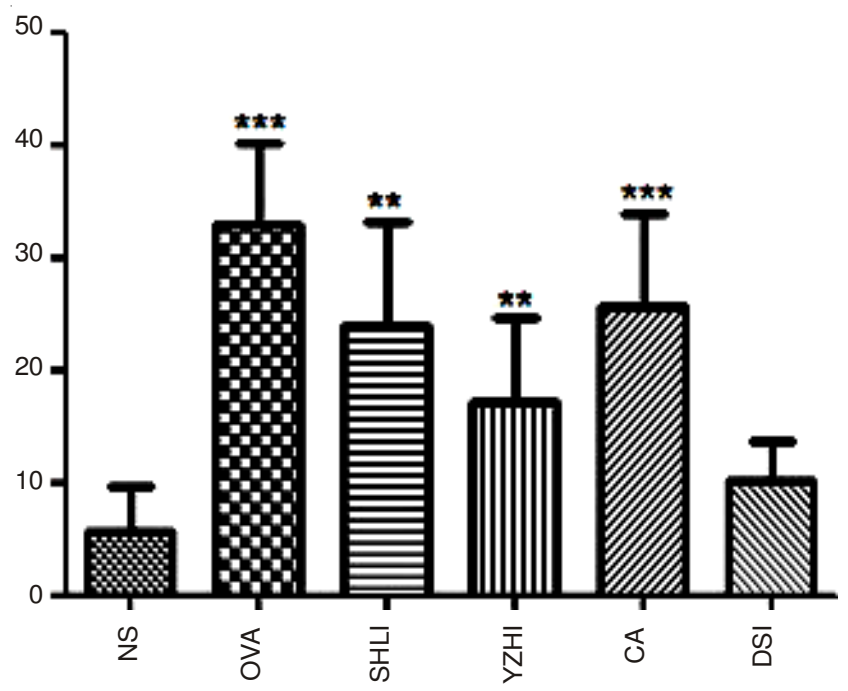

Fig. 3. Rising rate of $\beta$-hexosaminidase in allergic guinea pig plasma. Results are expressed as mean $\pm \mathrm{SD}(\mathrm{n}=6), * * \mathrm{P}<0.01$, ***P $<$ 0.001 vs. NS group

Determination of histamine in allergic guinea pig plasma: Histamine was measured with ELISA following the instruction of manufacturer. Determination results of histamine in allergic guinea pig plasma were shown in Fig. 4. The increasing rate of histamine in albumin chicken egg group, Shuanghuanglian group, Yinzhihuang group and chlorogenic acid group were statistically significant compared with normal saline group. However, it was not statistically significant in Dansheng group.

We herein found that the concentrations of 5-HT, $\beta$-hexosaminidase and histamine of Shuanghuanglian injection, Yinzhihuang injection, Dansheng injection, cholrogenic acid and albumin chicken egg solution in sensitized guinea pigs were increased in varying degrees. The rising rates of three indexes in Dansheng group were low and there was no significant difference between Dansheng group and normal saline group. However, the indexes of Yinzhihuang group, Shuanghuanglian group and cholrogenic acid group in sensitizied guinea pigs showed significant difference when compared with normal saline group. The Shuanghuanglian group had higher

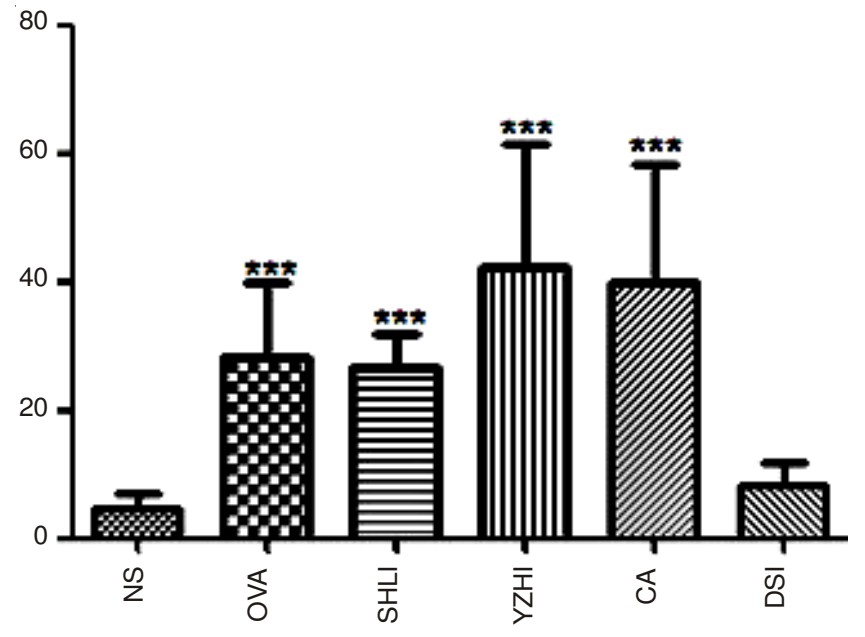

Fig. 4. Rising rate of Histamine in allergic guinea pig plasma. Results are expressed as mean \pm SD $(n=6), * * * P<0.001$ vs NS group

rising rate of 5-HT and $\beta$-hexosaminidase than Yinzhihuang group, but rising rate of histamine is lower. These notable results indicated that the target cells or the degree of sensitization might be different although they both showed the allergy symptoms. Moreover, the adverse reactions of herbal injections may be an allergic reaction or anaphylactoid reactions which could not be distinguished easily. Anaphylaxis and anaphylactoid reactions share a common symptom, but the mechanisms of them are different. Further investigations needed to be done to make it clear.

Shuanghuanglian injection is usually used for the treatment of respiratory infection and has been one of the top selling herbal medicine products over the last decade ${ }^{17}$. However, Shuanghuanglian injection often causes severe allergic reaction in the clinic ${ }^{18-20}$. Recent study had shown that, the cosolvent Tween-80 in herbal injection is the main inducement of allergic reaction and the medical shock ${ }^{21,22}$. However, there are no Tween-80 or other cosolvent in Shuanghuanglian injection and Yinzhihuang injection, the allergic reaction might caused by other substances. Both of Shuanghuanglian injection and Yinzhihuang injection contains honeysuckle and cholrogenic acid is considered to be the active compound in $\mathrm{it}^{23,24}$. Dansheng injection is composed of a single medicinal herb: Salvia miltiorrhiza which contains two primary components: fat-soluble two terpene quinones and water-soluble phenolic acids. From the different results between three injections, we speculate that the allergic reaction of Yinzhihuang injection and Shuangshuanglian injection is likely to be caused by cholrogenic acid. The batch number of Danshen injection in this study is 0806263 , which had not shown any allergic response reports in the clinic of China. Compared with other herbal injections, Dansheng injectionis more secure. Rare allergic reactions of Dansheng injection reported in clinical context might be mainly caused by the plant protein remained in the injection due to the differences of production processes and conditions between different manufacturers. The herbal injection is not as it is thought to be, which always cause a severe allergic reaction. In fact, no drug is without any side effects. As long as we establish a series of reasonable quality control specifications (QCS) of herbal injections, the application 
of herbal injections will not be limited. The detection of allergic reaction is the most important part of quality control of herbal injection. This study may provide a simple and reliable way to detect it.

\section{Conclusion}

In summary, the current results have shown that using 5-HT, $\beta$-hexosaminidase and histamine to evaluate the allergenicity of the herbal injection is reliable with high degree of accurancy. And cholrogenic acid might be one of the incentives of allergic reaction in Shuanghuanglian injection and Yinzhihuang injection.

\section{ACKNOWLEDGEMENTS}

This work was supported by the Program of State Key Laboratory of New-Tech for Chinese Medicine Pharmaceutical Process (No. SKL2010Z0403) and the Specialized Research Fund for the Doctoral Program of Higher Education of China (No.20123237110009) and the grant from the National Science Foundation of China (No. 30973972).

\section{REFERENCES}

1. Y.Z. Liang, P. Xie and K. Chan, J. Chromatogr. B, Biomed. Sci. Appl., 812, 53 (2004).

2. M.L. Willcox and G. Bodeker, BMJ, 329, 1156 (2004).

3. L. Jiabo, Mod. Tradit. Chin. Med. Mater. Med., 12, 497 (2010).

4. K.M. Ji, J.J. Chen, M. Li, Z.G. Liu, L.X. Xia, C.B. Wang, Z. Zhan and X. Wu, Regul. Toxicol. Pharmacol., 55, 134 (2009).

5. J. Hu, H. Shang, J. Li, J. Zhang, L. Zhang, W. Zheng, T. Li, B. Zhang and Y. Li, J. Evid. Based Med., 3, 37 (2010).
6. Z. Wang, D. Wang, Y. Sui, H. Cui and Y. Yu, J. Tradit. Chin. Med., 32, 641 (2012).

7. D. Dombrowicz, V. Flamand, I. Miyajima, J.V. Ravetch, S.J. Galli and J.P. Kinet, J. Clin. Invest., 99, 915 (1997).

8. I. Miyajima, D. Dombrowicz, T.R. Martin, J.V. Ravetch, J.P. Kinet and S.J. Galli, J. Clin. Invest., 99, 901 (1997).

9. Finkelman. R.T. Strait, S.C. Morris, M. Yang, X.-W. Qu and F.D. Finkelman, J. Allergy Clin. Immunol., 109, 658 (2002).

10. S.J. Galli, J. Allergy Clin. Immunol., 115, 571 (2005).

11. W. Jacoby, P.V. Cammarata, S. Findlay and S.H. Pincus, J. Invest. Dermatol., 83, 302 (1984).

12. D.D. Metcalfe, R.D. Peavy and A.M. Gilfillan, J. Allergy Clin. Immunol., 124, 639 (2009).

13. J.J. Hu, Y.M. Hou, Q. Zhang, H. Lei, Y. Wang and D. Wang, Zhongguo Zhong Yao Za Zhi, 36, 1860 (2011) (in Chinese.).

14. T.C. Theoharides, P.K. Bondy, N.D. Tsakalos and P.W. Askenase, Nature, 297, 229 (1982).

15. J. Zhang, Y.K. Li, L.D. Li, W. Hao and W.J. Yan, Chin. J. New Drugs, 17, 1494 (2008) (in Chinese.).

16. F. Huang, X. Zhang, L. Zhang, Q. Li, B. Ni, X.L. Zheng and A. Chen, Acta Pharmacol. Sin., 31, 849 (2010)

17. R. Han, J.X. Ye, L.H. Quan, C.Y. Liu, M. Yang and Y.H. Liao, J. Ethnopharmacol., 135, 522 (2011).

18. X. Chen, O.M.Z. Howard, X. Yang, L. Wang, J.J. Oppenheim and T. Krakauer, Life Sci., 70, 2897 (2002).

19. Z.G. Li, Y. Gao, H.S. Wang and Z.P. Liu, Asian Pac. J. Allergy Immunol., 28, 185 (2010).

20. T. Wu, H. Shang, Z. Bian, J. Zhang, T. Li, Y. Li and B. Zhang, J. Evid. Based Med., 3, 11 (2010).

21. L. Xia, W. Qing, Z. Lian, D. Yan and J. Yiping, Adv. Drug React. J., 3, 4 (2010).

22. T. Liu, Y. Zhao, C. Cao, R. Hao, C. Li and Y. Yi, Zhongguo Zhong Yao Za Zhi, 35, 1603 (2010) (in Chinese.).

23. Y. Ren, P. Zhang, D. Yan, J. Wang, X. Du and X. Xiao, J. Pharm. Biomed. Anal., 56, 436 (2011).

24. L. Liu, W. Jiang, L. Zhang, F. Li and Q. Zhang, J. Sep. Sci., 34, 1834 (2011). 